\title{
Fruit and vegetable intake decreased risk of ischaemic stroke
}

Joshipura KJ, Ascherio A, Manson JE, et al. Fruit and vegetable intake in relation to risk of ischemic stroke.JAMA 1999 Oct 6;282:1233-9.

QUESTION: Is increased fruit and vegetable intake associated with a reduction in ischaemic stroke?

\section{Design}

Population based cohort study of women in the Nurses' Health Study with 14 years of follow up, and men in the Health Professionals' Follow up Study with 8 years of follow up.

\section{Setting \\ USA.}

\section{Participants}

121700 women in the Nurses' Health Study who were 30-55 years of age in 1976 (baseline) and 51529 men in the Health Professionals' Follow up Study who were 40-75 years of age in 1986 (baseline). Exclusion criteria were $\geqslant 10$ non-responses to dietary questions for women or $\geqslant 70$ non-responses for men; implausible scores for total food intake; or previous cancer, diabetes, or cardiovascular disease. Data from 75596 women and 38683 men were eligible for analysis.

\section{Assessment of risk factors}

Participants completed postal questionnaires at baseline and every 2 years thereafter. Food frequency questionnaires gathered information on food and beverage intake and portion sizes. For analysis, participants were divided into 5 groups (quintiles) based on mean daily intakes of individual fruits, vegetables, and composite items of fruits or vegetables; the lowest quintile for each item was given a relative risk (RR) of 1.0. Data were adjusted for age; smoking; alcohol use; family history of myocardial infarction; body mass index; multivitamin, vitamin $\mathrm{E}$, and aspirin use; physical activity; hypertension; hypercholesterolaemia; total energy intake; time period; and, among women, postmenopausal hormone use.

\section{Main outcome measure}

Stroke, confirmed by blinded assessment of medical records.

\section{Main results}

During follow up, 670 cases of stroke were reported among women (366 ischaemic, 198 haemorrhagic, and 106 unknown types of stroke) and 317 cases were reported among men (204 ischaemic, 64 haemorrhagic, and 49 unknown types of stroke). The men and women in the top quintile of total fruit and vegetable intake (median 9.15 and 10.15 servings/d for men and women, respectively) had a lower risk of ischaemic stroke (RR 0.69, 95\% CI 0.52 to 0.92 ) when compared with those in the bottom quintile (median 2.54 and 2.93 servings/d for men and women, respectively). Lower risks of ischaemic stroke were associated with an increment of 1 serving/day (based on the median value for the quintile of intake) of fruits or vegetables (RR 0.94, CI 0.90 to 0.99,p for trend 0.01), all citrus fruits (RR 0.81, CI 0.68 to 0.96 ), citrus fruit juices (RR 0.75 , CI 0.61 to 0.93 ), cruciferous vegetables such as broccoli or cabbage (RR 0.68, CI 0.49 to 0.94 ), and green leafy vegetables (RR 0.79, CI 0.62 to 0.99). Risk of ischaemic stroke was not associated with intake of legumes (RR 1.09, CI 0.47 to 2.51 ) or potatoes (RR 1.21, CI 0.89 to 1.64 ).

\section{Conclusion}

Intake of fruit and vegetables, specifically citrus fruits and juice, cruciferous vegetables, and green leafy vegetables were associated with a lower risk of ischaemic stroke.

\section{COMMENTARY}

The evidence linking fruit and vegetable intake with specific health outcomes is sparse. The findings of this study by Joshipura et al, consistent with those of a previous study, showed that for every additional serving of fruit or vegetable consumed, a reduction in the risk of ischaemic stroke was gained. This effect was noted for every additional serving consumed, up to 6 servings. Cruciferous vegetables (eg, broccoli, cabbage, cauliflower, and Brussels sprouts), green leafy vegetables, citrus fruits, and vitamin C rich fruit and vegetables were associated with the greatest reduction in risk of stroke.

The data were from 2 large cohorts and the analysis was adjusted for potential effects of many factors. The generalisability of these data from nurses, dentists, veterinarians, pharmacists, and other health professionals to other groups is unknown. This population was relatively well educated and probably had better socioeconomic status and health, and a lower risk of mortality (including stroke mortality). ${ }^{2}$ In this study, those with the poorest diet had a median daily intake of about 2.5 servings of fruits and vegetables. Future studies that include people with very poor diets may reveal a stronger relation between diet and ischaemic stroke.

Stroke is a public health problem of significant magnitude that is preventable through lifestyle change. A primary prevention approach focused on encouraging the public to consume 5 servings of fruits and vegetables each day may reduce its prevalence. Food consumption, however, is linked to complex economic, social, cultural, and behavioural factors. Goals for education on nutrition should be directed towards individuals and communities with potential for promotion (eg, retail shops), and may also include service measures, including food assistance programmes; legislative or regulatory measures to ensure healthy diets in institutions and the workplace; and economic measures to foster workplace nutritional health promotion and coverage of nutritional counselling by healthcare insurers.

\section{Pamela A Ratner, RN, PhD} Assistant Professor

University of British Columbia Vancouver, British Columbia, Canada

1 Gillman MW, Cupples LA, Gagnon D, et al. Protective effect of fruits and vegetables on development of stroke in men. JAMA 1995;273:1113-7.

2 Kunst AE, del Rios M, Groenhof F, et al. Socioeconomic inequalities in stroke mortality among middle-aged men: an international overview. European Union Working Group on Socioeconomic Inequalities in Health. Stroke 1998;29:2285-91. 